\title{
Analyse et conception d'un coupleur coaxial rectangulaire à conducteurs internes circulaires par la méthode des éléments finis
}

\author{
Salima Seghier et NasrEddine Benahmed* \\ Université de Tlemcen, Faculté des sciences de l'ingénieur, B.P. 119, \\ Tlemcen 13000, Algérie
}

(Reçu le 05 Mars 2006, accepté le 16 Juillet 2006)

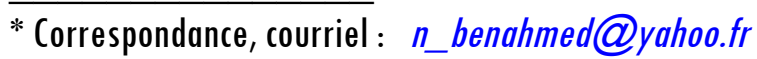

\section{Résumé}

Dans cet article nous présentons, d'une part l'analyse d'un coupleur coaxial rectangulaire à conducteurs internes circulaires ef d'autre part les résultats de conception pour un coupleur directif. L'analyse est basée sur une résolution numérique de l'équation de Laplace par la méthode des éléments finis. Cette technique performante est très utilisée pour la caractérisation des grandeurs électromagnétiques de système de lignes de forme très complexe et n'admettant pas une solution analytique exacte. La modélisation du coupleur consiste à la détermination des impédances caractéristiques des deux modes pair et impair $\left(Z_{0 e}, Z_{00}\right)$, du coefficient de couplage $(k)$ et des matrices d'inductances et de capacités ([L] et $[C])$.

L'outil numérique développé reste valable pour tout coupleur TEM de type symétrique.

Mots-clés : coupleur directif, coupleur coaxial rectangulaire, conducteur interne circulaire, paramètres électromagnétiques $Z_{o e}, Z_{00}, k,[L]$ et $[C]$, méthode des éléments finis.

\section{Abstract \\ Finite element analysis and design of a rectangular coaxial coupler with circular inners conductors}

In this paper, we present the analysis of rectangular coaxial coupled lines with circular inners conductors. This analysis is based on a numerical resolution of the Laplace's equation by the finite element method (FEM). This technique is adapted to study the complex configuration of the line's system which does not have simple analytical 
solution. The modelling of this structure consists in analysing the even and the odd mode characteristic impedances $\left(Z_{0 e}, Z_{00}\right)$, the coupling coefficient $(k)$ and the inductance and capacitance matrices ([L] and $[\mathrm{C}]$ matrices, respectively).

Also we present the results of a directional coupler design. The numerical model developed remains valid to all TEM and symmetrical couplers.

Keywords : directional coupler, rectangular coaxial coupler, circular inner conductor, electromagnetic parameters $Z_{o e}, Z_{o o}, k,[L]$ and $[C]$, finite element method (FEM).

\section{Introduction}

La caractérisation analytique du couplage entre deux lignes coaxiales rectangulaires à conducteurs internes circulaires est une tâche difficile; toutefois, des méthodes numériques peuvent résoudre ce problème. Pour atteindre cet objectif, il est nécessaire tout d'abord d'évaluer numériquement les impédances caractéristiques des modes pair et impair et ensuite le coefficient de couplage, les matrices d'inductances et de capacités et la réponse fréquentielle de la structure [1,2].

Dans ce présent travail, nous nous intéressons à la caractérisation numérique par la méthode des éléments finis des impédances caractéristiques des deux modes pair et impair, du coefficient de couplage, des matrices d'inductances et de capacités et de la réponse fréquentielle de deux lignes coaxiales rectangulaires et à conducteurs internes circulaires, couplées entre elles. En utilisant cette technique nous montrons l'influence des paramètres physiques $\left(\varepsilon_{\mathrm{r}}\right)$ et géométriques sur les caractéristiques électromagnétiques du type de coupleur analysé.

Enfin, nous présentons les résultats de conception d'un coupleur directif.

\section{Coupleur coaxial rectangulaire à conducteurs internes circulaires}

Récemment, un nouveau type de coupleur directif a été élaboré à partir de deux câbles coaxiaux, à blindage rectangulaire, couplés entre eux. Ce type de coupleur est très performant, vu sa grande directivité, son faible rapport d'ondes stationnaires, sa bonne isolation et son faible coût [3]. La section transversale d'un tel coupleur est présentée sur la Figure 1. 


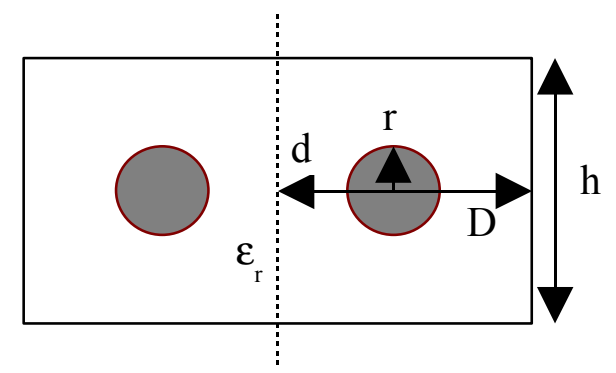

Figure 1 : Section transversale du coupleur rectangulaire à conducteurs internes circulaires

Cette Figure présente deux câbles coaxiaux à faible perte coupés et couplés entre eux. Chacun d'eux est constitué d'un conducteur interne de rayon $\left(r_{c}\right)$ et d'un conducteur externe (blindage) de hauteur (h) et de largeur (2D). Un diélectrique de permittivité relative $\left(\varepsilon_{\mathrm{r}}\right)$, règne à l'intérieur du câble. La profondeur de coupure (dite aussi de couplage) est représentée par (d) sur la section transversale et elle est définie par [3,4] :

$$
u=\frac{\operatorname{Cos}^{-1}\left(\frac{d}{D}\right)}{\operatorname{Cos}^{-1}\left(\frac{r_{c}}{D}\right)}
$$

Les propriétés électriques de ce coupleur, réalisé à base de lignes à faible perte et propageant le mode TEM, peuvent être décrites en termes d'impédances caractéristiques d'un mode pair $\left(Z_{0 e}\right)$ et d'un mode impair $\left(Z_{00}\right)$ et en termes de matrices $[L]$ et $[C][1,5,6]$.

$$
[L]=\left[\begin{array}{cc}
L_{0} & M \\
M & L_{0}
\end{array}\right] ;[C]=\left[\begin{array}{cc}
C_{o} & -\gamma \\
-\gamma & C_{o}
\end{array}\right]
$$

où :

$L_{0}$ et $C_{0}$ sont respectivement l'inductance et la capacité propre de la ligne isolée; $M$ et $\gamma$ sont respectivement l'inductance mutuelle et la capacité de couplage du système de lignes couplées.

\section{Solution numérique}

L'étude de la structure présentée sur la Figure 1, dans le domaine électrostatique, est basée sur la résolution de l'équation de Laplace en 2D pour les deux modes (pair et impair):

$$
\operatorname{div}\left[\operatorname{gra} d_{t} V(x, y)\right]=0
$$


Mode pair :

$\mathrm{V}=1 \mathrm{~V}$ sur les deux conducteurs internes.

$\mathrm{V}=0 \mathrm{~V}$ sur le blindage.

Mode impair :

$V=1 \mathrm{~V}$ sur l'un des deux conducteurs internes.

$V=-1$ V sur l'autre conducteur interne.

$\mathrm{V}=0 \mathrm{~V}$ sur le blindage.

En introduisant une formulation variationnelle [7] associée à l'équation (3), nous obtenons :

$\iint_{\Omega}\left(\operatorname{gra} d_{t} V(x, y)\right) \cdot\left(\operatorname{gra} d_{t} V^{*}(x, y)\right) d x d y=0$

où $\Omega$ est le domaine de maillage de la structure.

L'équation (4) est ensuite discrétisée sur chaque élément triangulaire du maillage, par approximation du potentiel V par des polynômes d'interpolation de Lagrange. Cette opération conduit à un système linéaire élémentaire de la forme :

$[K]^{e}\{V\}^{e}=\{0\}$

où $[K]^{e}$ est une matrice élémentaire et $\{V\}^{e}$ est le vecteur solution des potentiels scalaires au niveau des noeuds de l'élément triangulaire considéré.

L'assemblage de tous ces systèmes élémentaires conduit à un système global dont la résolution par des techniques numériques adaptées donne la distribution du potentiel scalaire aux différents nœuds de maillage de la structure.

Quand le potentiel $V$ est déterminé, nous calculons tout d'abord les impédances caractéristiques des modes pair et impair et ensuite le coefficient de couplage, les matrices d'inductance et de capacité et la réponse fréquentielle de la structure.

\section{3-1. Détermination des impédances caractéristiques des modes pair et impair}

La théorie des lignes de transmission sans perte nous permet de déterminer le champ électrique et magnétique à partir du potentiel V. L'énergie électrique $W_{\mathrm{em}}$ emmagasinée dans la structure est calculée à partir du champ électrique et toutes les impédances caractéristiques (des deux modes) sont déduites facilement à partir de l'énergie électrique $W_{\text {em. }}$. Par conséquent, il est impératif de calculer le potentiel $V$ avec une grande précision.

La procédure de calcul pour un mode donné est la suivante ; on peut déduire du potentiel scalaire $V$, le champ électrique transverse par simple dérivation sachant que : 
$\vec{E}_{t}=-\operatorname{gra} d_{t}(V)$

La structure emmagasine une énergie électrique moyenne qu'on peut déduire du champ électrique transverse comme suit :

$\bar{W}_{e m}=\frac{1}{4} \iint \varepsilon_{0} \varepsilon_{r} \vec{E}_{t} \cdot \vec{E}_{t}^{*} d x d y$

La capacité linéique est déduite directement de l'énergie électrique moyenne, soit :

$C=\frac{4 \bar{W}_{e m}}{\left(V_{1}-V_{2}\right)^{2}} \quad$ en $(\mathrm{F} / \mathrm{m})$

où $V_{1}$ et $V_{2}$ sont les conditions d'excitations de la structure.

L'impédance caractéristique est calculée à partir de la relation suivante :

$Z_{c}=\frac{1}{v_{\varphi} C}$ en $(\Omega)$

où $\quad v_{\varphi}=\frac{3.10^{8}}{\sqrt{\varepsilon_{r}}}(\mathrm{~m} / \mathrm{s})$ est la vitesse de phase.

L'inductance linéique se calcule à partir de l'impédance caractéristique et de la capacité linéique, soit :

$L^{\prime}=Z_{c}^{2} C$ en $(H / m)$

Cette procédure est encore utilisée pour le calcul numérique des paramètres linéiques $\left(L_{0}\right.$ et $\left.C_{0}\right)$ de la ligne coaxiale rectangulaire isolée.

\section{3-2. Détermination du coefficient de couplage}

Les valeurs des impédances caractéristiques $Z_{\text {oe }}$ et $Z_{00}$, respectivement associées aux modes pair et impair, étant alors connues, nous calculons le coefficient de couplage $k$ en utilisant la relation suivante :

$k=\frac{Z_{o e}-Z_{o o}}{Z_{o e}+Z_{o o}}$

\section{3-3. Détermination des matrices d'inductance et de capacité}

Une fois le coefficient de couplage $k$ déterminé, on peut déduire les valeurs de $\gamma$ (capacité de couplage), et de $M$ (inductance mutuelle), en écrivant : 
$k=\frac{\gamma}{C_{0}}=\frac{M}{L_{0}}$

où $\mathrm{L}_{0}$ et $\mathrm{C}_{0}$ sont les paramètres linéiques de la ligne coaxiale rectangulaire isolée calculés numériquement par la MEF

Ce qui permet de déterminer les matrices [L] et [C] ( relation (2)) pour le système formé des deux lignes en question.

\section{3-4. Méthode de conception et réponse fréquentielle du coupleur micro-onde directif}

La Figure 2 présente la structure d'un coupleur coaxial rectangulaire à conducteurs internes circulaires, de longueur ( I ) et chargé par des impédances $Z_{L}$ de valeurs $50 \Omega$.

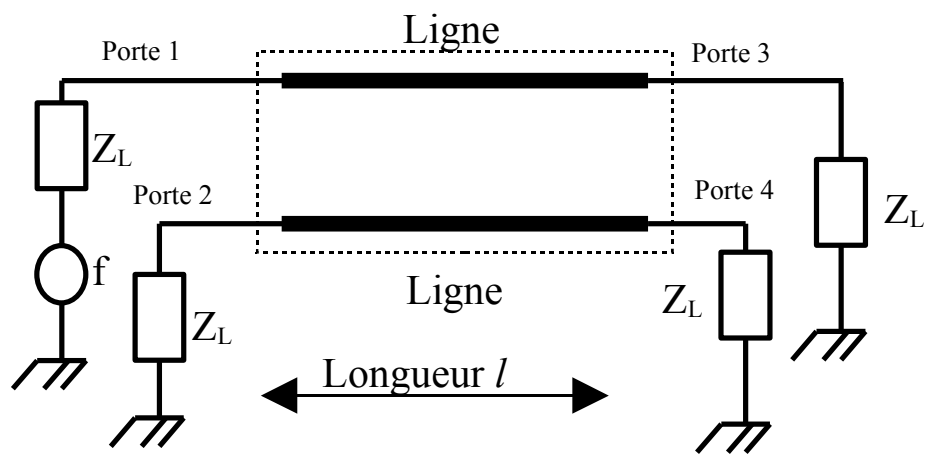

Figure 2 : Schéma équivalent du coupleur coaxial rectangulaire à conducteurs internes circulaires.

La conception de ce type de coupleur se fait systématiquement pour une longueur $l=\frac{\lambda}{4}=\frac{v_{\varphi}}{4 . f}$, soit des lignes couplées $l / 4$ d'onde, où f est la fréquence de travail.

Pour que le système de lignes couplées soit adapté au niveau de ses quatre portes, il est impératif que son impédance caractéristique $Z_{c}$ définie par : $Z_{c}=\sqrt{Z_{o e} Z_{o o}}$ soit égale à l'impédance de charge $Z_{L}$. Comme $Z_{c}$ est aussi l'impédance caractéristique de la ligne coaxiale rectangulaire isolée (Figure 3), alors on peut déterminer la valeur du rapport $\mathrm{D} / \mathrm{r}_{\mathrm{c}}$ pour $\mathrm{Z}_{\mathrm{c}}=\mathrm{Z}_{\mathrm{L}}=50 \Omega$, en utilisant la relation suivante, ov̀ $(\mathrm{h}=2 \mathrm{D})[8]$ :

$$
Z_{c} \approx \frac{60}{\sqrt{\varepsilon_{r}}} \log \frac{1.078 D}{r_{c}}
$$




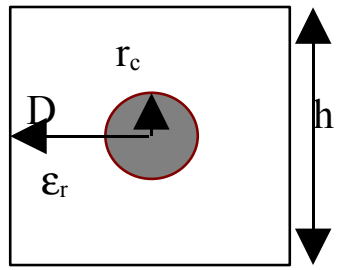

Figure 3 : Section transversale de la ligne coaxiale rectangulaire isolée.

Pour un milieu diélectrique de permittivité relative $\varepsilon_{\mathrm{r}}=2.03$, la valeur du rapport $\mathrm{D} / \mathrm{r}_{\mathrm{c}}$ assurant une impédance caractéristique $Z_{c}=50 \Omega$, est de 3.03 .

Une fois le coefficient de couplage déterminé (numériquement par la MEF) ainsi que les impédances caractéristiques des modes pair et impair, nous pourrons trouver pour un couplage donné, la valeur de la profondeur de coupure (u) du système de lignes coupées et couplées.

Ainsi, tous les paramètres géométriques et physiques sont obtenus. II ne restera qu'à trouver les valeurs des éléments des matrices d'inductances $[L]$ et de capacités $[C]$ du coupleur pour pouvoir évaluer les coefficients de couplage $\left(S_{12}\right)$ et d'isolation $\left(S_{14}\right)$ en utilisant un modèle numérique adapté [9]. Cette évaluation permet de décider si les contraintes permettent la réalisation expérimentale du coupleur ou non.

\section{Résultats numériques}

Sur la base de la théorie présentée ci dessus, nous avons établi un programme permettant le calcul des impédances caractéristiques des modes pair et impair, du coefficient de couplage, des matrices d'inductance et de capacité et de la réponse fréquentielle du système de lignes couplées en fonction de ses caractéristiques physiques et géométriques.

Pour valider nos résultats numériques obtenus par la MEF, nous avons analysé la structure présentée sur la Figure 1 , où :

$-\mathrm{D} / \mathrm{r}_{\mathrm{c}}=3.03$,

$-\mathrm{h}=2 \mathrm{D}$,

$-\varepsilon_{\mathrm{r}}=2.03$.

Les Figures 4 et 5 illustrent respectivement le maillage de la section transversale du coupleur et les équipotentielles de ses deux modes pair et impair après résolution de l'équation de Laplace. 


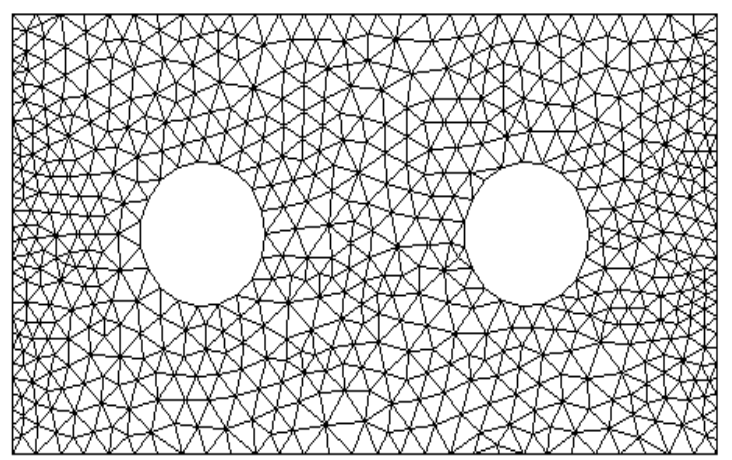

Figure 4 : Maillage de la structure.

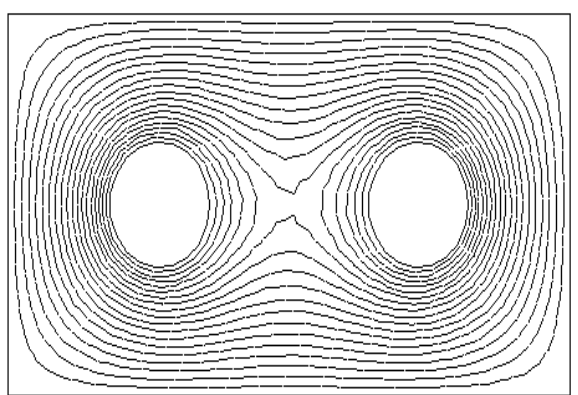

Mode pair

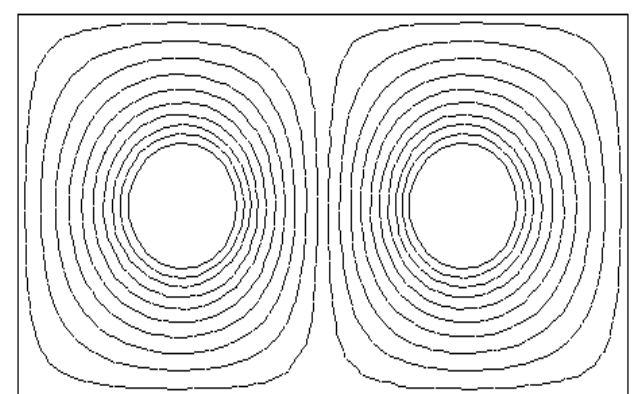

Mode impair

Figure 5 : Lignes équipotentielles des modes pair et impair.

Pour pouvoir vérifier le bien fondé de nos calculs numériques, nous nous sommes intéressés en premier lieu à l'influence de la profondeur de coupure (u) sur les impédances caractéristiques des modes pair et impair. Une comparaison entre nos résultats numériques obtenus par la méthode des éléments finis (MEF) et ceux trouvés par la méthode des moments (MoM) [10] est montrée sur les graphes de la Figure 6.

De ces graphes, il apparaît clairement une très bonne cohérence entre les deux méthodes numériques utilisées. De plus, on constate que pour toute valeur de la profondeur de coupure (u) comprise entre 0.05 et 1 , l'impédance caractéristique du système de lignes couplées $Z_{\mathrm{c}}\left(Z_{c}=\sqrt{Z_{o e} Z_{o o}}\right)$ est aux alentours de $50 \Omega$, ce qui permet d'obtenir une très bonne adaptation aux différentes portes du coupleur. 


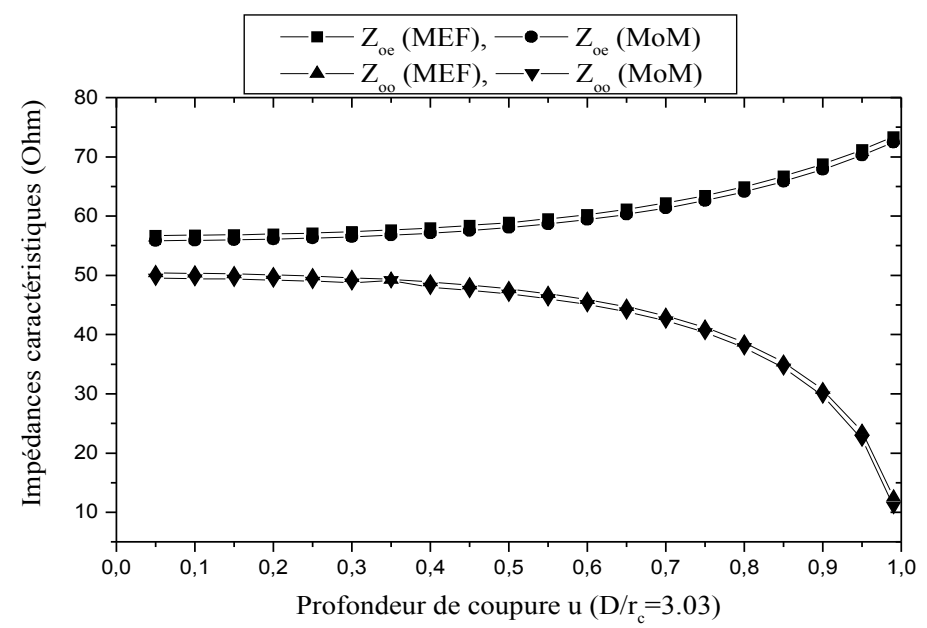

Figure 6 : Effet de la profondeur de couplage sur les impédances caractéristiques des modes pair et impair.

Nous nous sommes intéressés ensuite à l'influence de la profondeur de coupure (u) sur le coefficient de couplage. Cette influence est montrée sur le graphe de la Figure suivante :

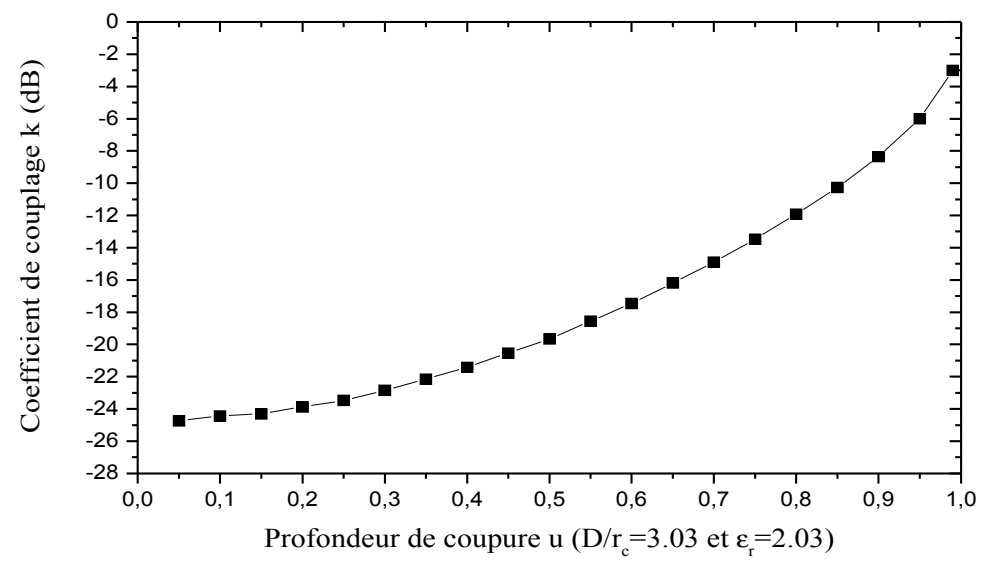

Figure 7 : Influence de la profondeur de coupure (u) sur le coefficient de couplage (k).

A partir de ce graphe on peut déterminer la valeur de la profondeur de coupure (U) correspondante à un couplage, par exemple, de $20 \mathrm{~dB}$. Cette valeur est d'environ 0.45 . 
Il est intéressant d'étudier la dépendance entre la profondeur de coupure (U) et les éléments des matrices d'inductances $[\mathrm{L}]$ et de capacités $[C]$ pour les mêmes paramètres géométriques et physiques ci dessus. Nous montrons cette dépendance sur les graphes des Figures 8 à 11 :

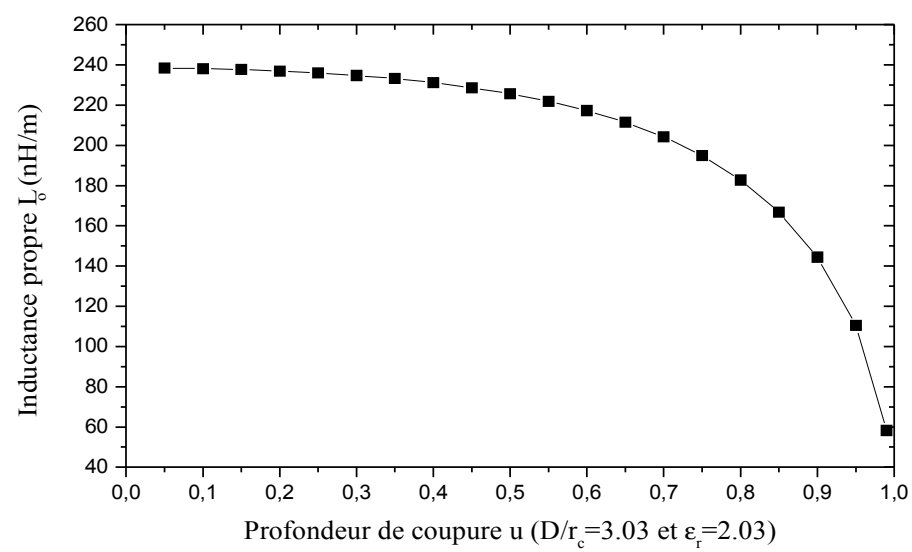

Figure 8 : Influence de la profondeur de coupure $(u)$ sur l'inductance propre $\left(L_{0}\right)$.

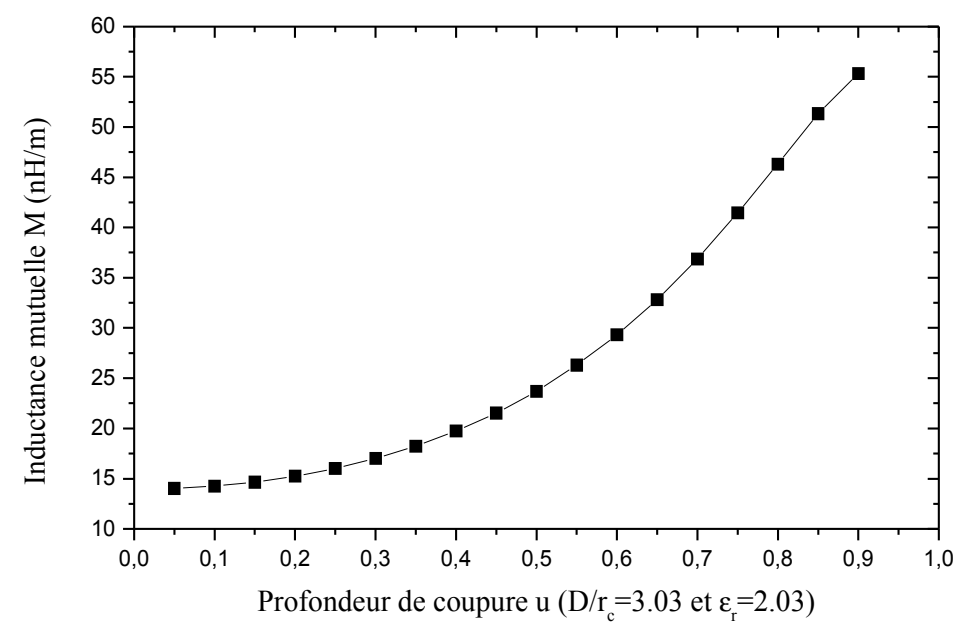

Figure 9 : Influence de la profondeur de coupure (U) sur l'inductance mutuelle (M). 


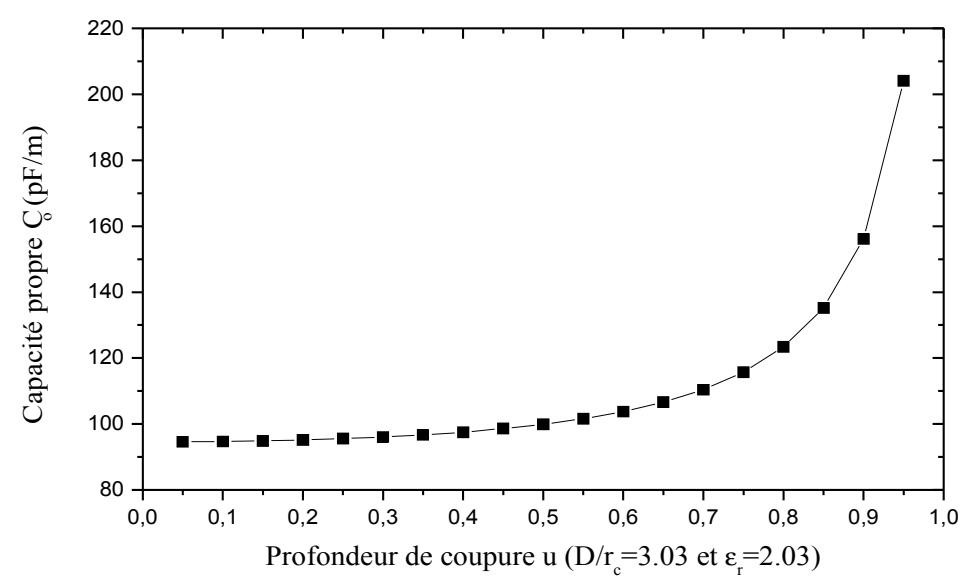

Figure 10 : Influence de la profondeur de coupure $(U)$ sur la capacité propre $\left(C_{0}\right)$.

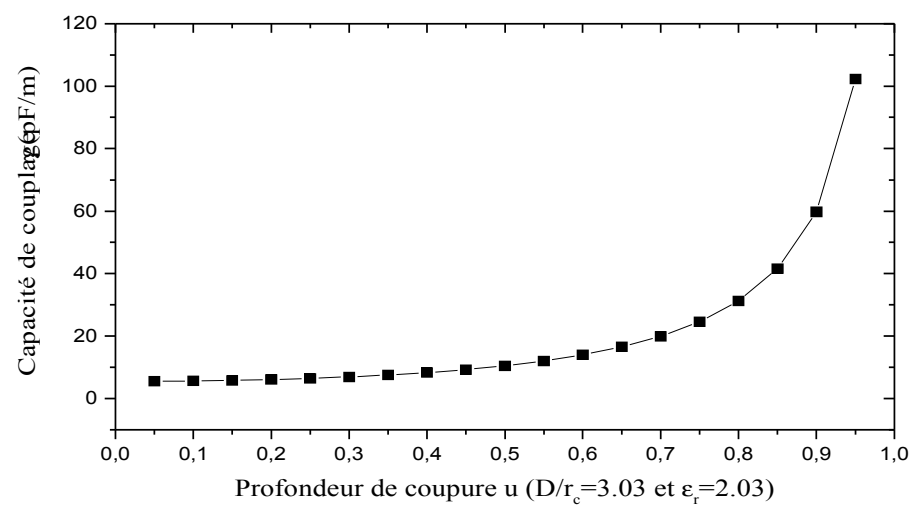

Figure 11 : Influence de la profondeur de coupure (u) sur la capacité de couplage ( $\gamma)$.

Il est intéressant de signaler que les graphes des Figures $7 \grave{a} 11$ sont nécessaires pour la conception de coupleurs coaxiaux rectangulaires à conducteurs internes circulaires d'impédance $50 \Omega$ ayant un couplage compris entre $3 \mathrm{~dB}$ et $24.73 \mathrm{~dB}$ pour une profondeur de coupure comprise entre 0.05 et 0.99 . Par conséquent ce type de coupleur symétrique et homogène permet d'obtenir des couplages forts [ $3 ; 24.74] \mathrm{dB}$ ce qui n'est pas le cas, par exemple, pour les coupleurs asymétriques et inhomogènes (coaxmicrostrip, coax-stripline) analysés dans les références [2] et [11] et permettant d'obtenir de faibles couplages $[11 ; 55] \mathrm{dB}$. 


\section{Conception du coupleur directif}

Pour concevoir un coupleur coaxial rectangulaire à conducteurs internes circulaires, directif et fonctionnant dans la plage de fréquences $[0.6-10] \mathrm{GHz}$ ayant :

- Impédance caractéristique : $\quad Z_{c}=50 \Omega$

- Coefficient de couplage désiré : $k(d B)=20 \mathrm{~dB}$

- Fréquence de travail : $\quad f=5 \mathrm{GHz}$

Alors d'après les résultats numériques que nous avons obtenus précédemment, les paramètres géométriques et physiques correspondants sont :

$-\mathrm{D} / \mathrm{r}_{\mathrm{c}}=3.03$

$-\mathrm{h}=2 \mathrm{D}$

- Profondeur de couplage : $\quad u=0.45$

- Longueur du coupleur : $\quad I=10.5 \mathrm{~mm}$

- Constante diélectrique : $\quad \varepsilon_{\mathrm{r}}=2.03$

Les graphes des Figures $\mathbf{6}, \boldsymbol{8} \dot{\boldsymbol{a}} \boldsymbol{l l}$ permettent de trouver les valeurs correspondantes des paramètres électromagnétiques du coupleur coaxial rectangulaire à conducteurs internes circulaires suivants :

- Impédances caractéristiques : $Z_{00}=58.38 \Omega ; Z_{00}=48.33 \Omega$

- Matrice d'inductances :

$$
[L]=\left[\begin{array}{ll}
233.761 & 20.698 \\
20.698 & 233.761
\end{array}\right]\left(\frac{n H}{m}\right)
$$

- Matrice de capacités :

$$
[C]=\left[\begin{array}{cc}
98.63 & -9.28 \\
-9.28 & 98.63
\end{array}\right]\left(\frac{p F}{m}\right)
$$

La connaissance des valeurs des éléments des matrices d'inductances [L] et de capacités [C] du coupleur nous permet d'évaluer les coefficients de couplage $\left(S_{12}\right)$ et d'isolation $\left(S_{14}\right)$ en utilisant un modèle numérique adapté [9].

Enfin, pour pouvoir décider si les paramètres géométriques et physiques $\left(D / \mathrm{r}_{\mathrm{c}}=3.03\right.$, $\mathrm{u}=0.45, \mathrm{I}=10.5 \mathrm{~mm}, \varepsilon_{\mathrm{r}}=2.03$ ) permettent la réalisation expérimentale du coupleur fonctionnant dans la plage de fréquence $[0.6-10] \mathrm{GHz}$ ou non, nous avons montré dans les graphes de la Figure 12 la variation fréquentielle des coefficients de couplage $\left(S_{12}\right)$ et d'isolation $\left(S_{14}\right)$. 


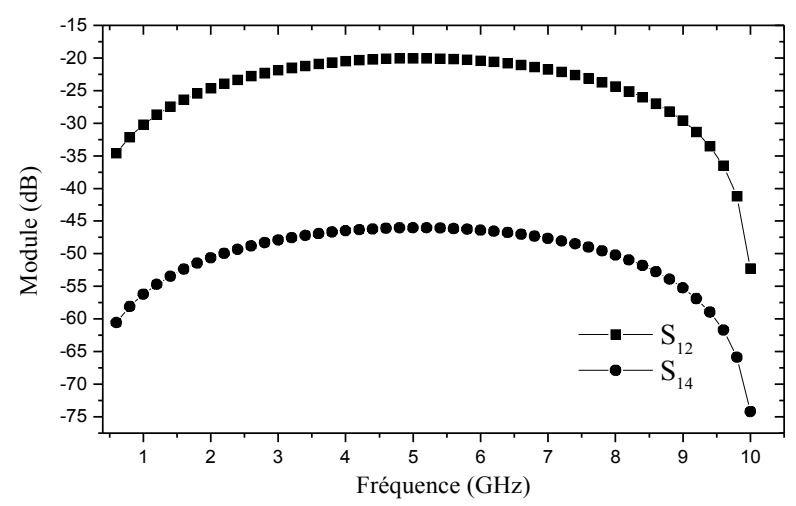

Figure 12 : Réponse du coupleur.

La Figure 12 montre que le couplage désiré de $20 \mathrm{~dB}$ est obtenu à la fréquence de travail $(f=5 \mathrm{GHz})$. Dans la plage de fréquence $[3,7] \mathrm{GHz}$ le couplage est compris entre $20 \mathrm{~dB}$ et $22 \mathrm{~dB}$ alors que l'isolation est comprise entre $46.02 \mathrm{~dB}$ et $47.66 \mathrm{~dB}$ ce qui assure au coupleur un minimum de directivité de $25 \mathrm{~dB}$ environ.

Ces résultats obtenus montrent clairement que les paramètres géométriques et physiques $\left(\mathrm{D} / \mathrm{r}_{\mathrm{c}}=3.03, \mathrm{u}=0.45, \mathrm{I}=10.5 \mathrm{~mm}, \varepsilon_{\mathrm{r}}=2.03\right)$ trouvés lors de la conception permettent effectivement la réalisation expérimentale du coupleur pour fonctionner dans la plage de fréquence $[3,7] \mathrm{GHz}$ avec un couplage et une directivité d'environ respectivement $20 \mathrm{~dB}$ et $25 \mathrm{~dB}$.

\section{Conclusion}

Le présent travail a consisté à réaliser par voie de simulation un coupleur micro-onde directif de $20 \mathrm{~dB}$ à $5 \mathrm{GHz}$ en utilisant deux lignes coaxiales rectangulaires et à conducteurs internes circulaires, couplées entre elles. Pour atteindre cet objectif, il a fallu déterminer les paramètres géométriques et électromagnétiques du système de lignes utilisé. Or aux fréquences du domaine $[0.6-10] \mathrm{GHz}$, le problème s'approxime par la résolution de l'équation de Laplace. Sa résolution à l'aide de la méthode des éléments finis a permis la détermination des impédances caractéristiques des modes pair et impair, du coefficient de couplage et des matrices d'inductances et de capacités. A travers les simulations que nous avons menées, nous constatons que l'écart est faible entre nos résultats numériques et ceux trouvés par la méthode des moments.

L'ensemble des courbes présentées dans ce travail, sous l'influence de la profondeur de coupure ou de couplage est nécessaire pour la conception de coupleurs directifs utilisant 
des lignes coaxiales rectangulaires et à conducteurs internes circulaires d'impédances $50 \Omega$ et de couplage compris entre $3 \mathrm{~dB}$ et environ $25 \mathrm{~dB}$. Ces courbes ainsi que celles de la réponse fréquentielle du coupleur témoignent de l'intérêt du modèle numérique développé qui reste valable pour tout coupleur TEM de type symétrique.

\section{Références}

[1] - N. Ben Ahmed and M. Feham, Microwaves \& RF, 45 (3) (2006) 86-92.

[2] - A. Sawicki, Microwave Journal, 48 (11) (2005) 106-111.

[3] - N. Ben Ahmed and S. Seghier, Microwave Journal, 49 (08) (2006) 164-174.

[4] - H. An, T. Wang, R.G. Bossio and K. Wu, IEEE Proceedings, (1995)

[5] - V. Teppati, IEEE Transactions on microwave theory and techniques, 50 (10) (2002) 2339-2345.

[6] - V. Teppati, IEEE Microwave \& Wireless Components Letters, 13 (4) (2003) 152-154.

[7] - G. Dhatt and G. Touzot, "Une présentation de la méthode des éléments finis", (1984), Collection Université de Compiègne, France.

[8] - E. Roubine, "Lignes et antennes", (1954), Edition de la revue d'optique, France.

[9] - A.R. Djordjevic, D.Darco, M.C. Goran and T.K. Sarkan, "Circuit Analysis Models for Multiconductors Transmission Lines", (1997), Artech House Inc.

[10] - A.R. Djordjevic, M.B. Bazdar, T.K. Sarkan and R.F. Harrington, "LINPAR for Windows: Matrix Parameters for Multiconductor Transmission Lines, Software and User's Manual", (1999), Artech House Inc.

[11] - N. Ben Ahmed and S. Seghier, Microwaves \& RF, 45 (10) (2006) 90-98. 